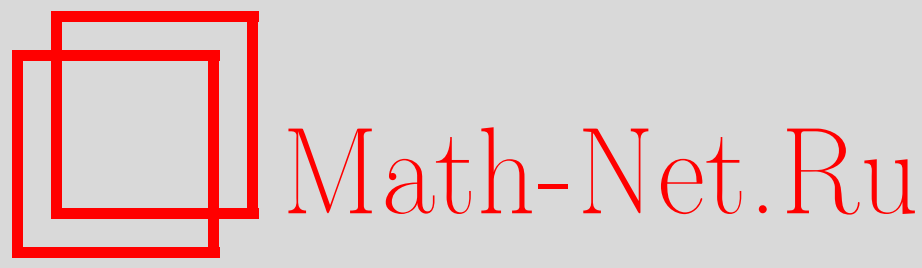

И. Я. Арефьева, Ультрарелятивистский адрон как космическая мембрана в моделях ТэВ-ной гравитации, TMФ, 2011, том 166, номер 3, 443-451

DOI: https://doi.org/10.4213/tmf6621

Использование Общероссийского математического портала Math-Net.Ru подразумевает, что вы прочитали и согласны с пользовательским соглашением http://www.mathnet.ru/rus/agreement

Параметры загрузки:

IP: 54.80 .97 .219

26 апреля 2023 г., 13:48:44

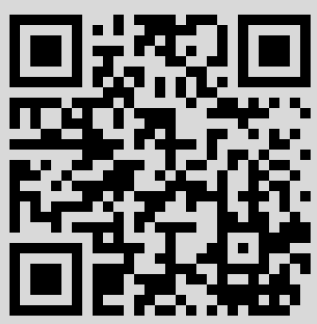




\title{
ФИЗИКА
}

Том 166, № 3

март, 2011

(C) 2011 г.

И. Я. Арефьева*

\section{УЛЬТРАРЕЛЯТИВИСТСКИЙ АДРОН КАК КОСМИЧЕСКАЯ МЕМБРАНА В МОДЕЛЯХ ТЭВ-НОЙ ГРАВИТАЦИИ}

\begin{abstract}
Используется представление об ультрарелятивистском адроне, движущемся по бране, вложенной в многомерное пространство-время, как о мембране, распространяющейся по 3-бране. При адронных размерах, бо́льших радиуса компактификации и характерных масштабов гравитационного взаимодействия, такую мембрану можно считать космической в том смысле, что она простирается бесконечно далеко. В пятимерном пространстве-времени космическая мембрана создает топологический дефект, аналогичный топологическому дефекту, который создает космическая струна в четырехмерном пространстве-времени. Такое представление позволяет учесть эффект влияния массы адрона на гравитационное взаимодействие партонов, входящих в состав сталкивающиеся адронов.
\end{abstract}

Ключевые слова: космическая мембрана, ТэВ-ная гравитация, высшие измерения.

\section{1. ВВЕДЕНИЕ}

В рамках стандартной теории элементарных частиц гравитационное взаимодействие между частицами пренебрежимо мало. Однако в рамках сценария ТэВ-ной гравитации [1], [2] гравитационное взаимодействие вносит существенный вклад во взаимодействие между элементарными частицами. Этот сценарий предполагает, что наш четырехмерный мир реализуется на бране [3], вложенной в многомерное пространство-время, и в многомерном пространстве-времени действует сильная гравитация с характерным масштабом взаимодействия порядка характерного масштаба стандартной модели 1 ТэВ. Гипотеза о существовании сильной гравитации приводит к предсказанию новых интересных явлений. В частности, в рамках такого сценария при столкновении частиц с энергией выше нескольких ТэВ [4], [5] (см. также ссылки в работе [6]) возможно образование черных дыр. В свою очередь, элементарные частицы могут создавать в рамках этого сценария достаточно сильное многомерное гравитационное поле, внося существенные изменения в характерный для выбранной модели гравитационный фон в многомерном пространстве-времени.

* Математический институт им. В. А. Стеклова РАН, Москва, Россия. E-mail: arefeva@mi.ras.ru 


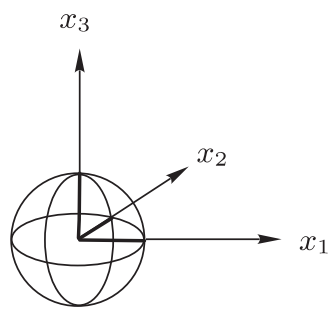

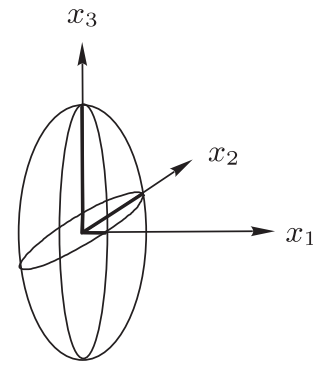

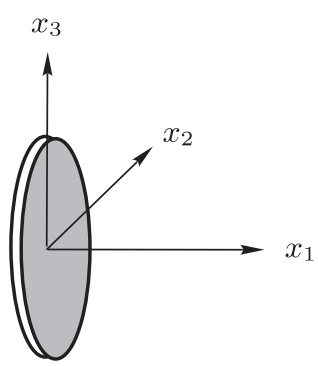

б

Рис. 1. Уплощение движущегося адрона в лабораторной системе отсчета (а). Схематическое представление ультрарелятивистского адрона как плоского диска (б).

Одним из простейших примеров такого изменения гравитационного фона является появление дефицита угла в пятимерной модели за счет материальной 2-браны (мембраны) [7]. Этот эффект является аналогом возникновения дефицита угла в четырехмерной модели за счет космической струны [8], который в свою очередь обусловлен дефицитом угла, создаваемым точечной частицей в трехмерной модели гравитации [9].

Можно рассмотреть также модель с анизотропной компактификацией на анизотропный тор, в которой размеры $n-1$ измерений существенно меньше одной выделенной дополнительной размерности. В этом случае можно добиться того, что $M_{\mathrm{Pl}, D} \sim 1$ ТэВ и при этом $M_{\mathrm{Pl}, 5} \sim 10^{3}$ ТэВ. Качественное поведение в этой модели такое же, как и в пятимерной "игрушечной" модели с $M_{\mathrm{Pl}, 5 \text { toy }} \sim 10^{3}$ ТэВ. Имея в виду дальнейшее рассмотрение в нетривиальном пятимерном пространстве-времени, в настоящей работе мы в основном исследуем случай плоского пространства-времени. В работе [7] показано, что космическая мембрана дает поправки к гравитационному эйкональному рассеянию. Здесь мы покажем, что адронная мембрана открывает новые каналы распада частиц, запрещенные в случае отсутствия топологического дефекта.

Статья организована следующим образом. В разделе 2 напоминаются основные предположения, ведущие к возможности существования космической мембраны, дана оценка пятимерной эффективной массы Планка в анизотропной модели ТэВ-ной гравитации и приведен вид метрик с коническими сингулярностями в пространствах Минковского и анти-де Ситтера. В разделе 3 приведены оценки сечений распада частиц на такой мембране.

\section{2. ОСНОВНЫЕ ПРЕДПОЛОЖЕНИЯ И ОБОЗНАЧЕНИЯ}

2.1. Модель ультрарелятивистского адрона. Если рассматривать ультрарелятивистский адрон в системе покоя как шарик, то в лабораторной системе отсчета он представляется как шарик, сплюснутый до достаточно тонкого диска (см. рис. 1). Этот диск мы описываем как движущуюся мембрану. Диаметр мембраны имеет порядок характерных размеров адрона. 


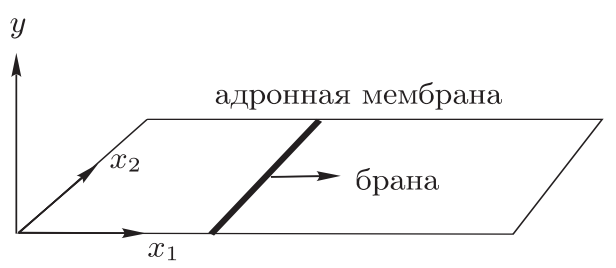

Рис. 2. Схематическое представление ультрарелятивистского адрона как мембраны, движущейся внутри 3-браны. Координата $x_{3}$ опущена, и адрон представлен как стержень, движущийся по поверхности 2-браны.

Если имеются $n$ дополнительных измерений, то они недоступны для адронов, и адронная мембрана не может простираться в эти измерения. Таким образом, рассматривая ультрарелятивистский адрон, движущийся по 3-бране, вложенной в $(4+n)$-мерное пространство-время, мы получаем картину, в которой двумерная адронная мембрана распространяется в 3-бране, вложенной в $(4+n)$-мерное пространство-время (см. рис. 2).

2.2. Метрики с коническими особенностями. Легко получить явные решения уравнений Эйнштейна в случае космической мембраны в пятимерном плоском пространстве-времени. Напомним, что в $(2+1)$-мерном пространстве решения уравнений Эйнштейна с точечными массами дают метрику, которая всюду плоская, однако имеет конические особенности, расположенные в тех точках, где находятся точечные массы. В этом случае дефицит угла конической особенности пропорционален массе [9]. В $(3+1)$-мерном пространстве с равномерным расположением масс вдоль плоского стержня (струны) решение уравнений Эйнштейна также представляет собой почти всюду плоское пространство-время с особенностью конического типа, причем особенность находится на мировом листе струны. В этом случае возникает дефицит угла между двумерными плоскостями, он равен плотности массы струны $\mu$, т.е. массе струны на единицу длины. В $(4+1)$-мерном пространстве существует решение с особенностью на мировом листе мембраны и дефицит угла возникает между трехмерными плоскостями. Эти мембраны, так же как точки и струны, в $(2+1)$-мерном и в $(3+1)$-мерном пространствах могут двигаться. Кроме того, благодаря тому, что известны решения для точечных масс в трехмерном пространстве анти-де Ситтера [10], мы знаем решения и для космической струны в четырехмерном пространстве анти-де Ситтера, а также и для космической мембраны в пятимерном пространстве анти-де Ситтера. Последний случай и соответствует модели Рэндалл-Сундрума RS2 [11]. Плоский пятимерный случай с 3-браной соответствует модели Аркани-Хамеда-Димопулоса-Двали ADD [1] и модели Двали-Габададзе-Поррати [12]. В плоском случае рассмотрение проще, и, несмотря на то что пятимерная модель ADD феноменологически неприемлема, для простоты мы рассмотрим в основном этот случай.

2.2.1. Метрика в модели ADD с конической особенностью. В модели ADD метрика в многомерном объеме плоская:

$$
d s^{2}=-d t^{2}+d \vec{x}_{\perp}^{2}+d \rho^{2}+\rho^{2} d \Omega^{2}, \quad \rho^{2}=\sum_{1}^{n} y_{i}^{2}+z^{2}, \quad \vec{x}_{\perp}=\left(x_{1}, x_{2}\right),
$$


где $x_{1}, x_{2}, y_{i}, z$ - координаты в многомерном пространстве-времени, $d \Omega^{2}$ - метрика на единичной сфере $S^{n}$. Однако наличие адронной мембраны обусловливает нетривиальный фон. Мы знаем этот фон явно в случае $n=1$. В этом случае метрика остается локально плоской, $d \Omega^{2}=d \phi^{2}$ и адронная мембрана производит только коническую особенность, т.е. угол меняется в области $0<\phi<\alpha$. Угол $\alpha$ определяет дефицит угла $\delta$ :

$$
\alpha=2 \pi-\delta
$$

где

$$
\delta=8 \pi G_{5} \frac{m_{\mathrm{h}}}{S_{\mathrm{h}}}=\frac{32}{M_{\mathrm{Pl}, 5}^{3}} \frac{m_{\mathrm{h}}}{l_{\mathrm{h}}^{2}},
$$

$m_{\mathrm{h}}$ и $l_{\mathrm{h}}$ - масса и размер адрона, $S_{\mathrm{h}}=\pi l_{\mathrm{h}}^{2} / 4$. Вершина конуса находится на бране.

Мы видим, что дефицит угла пропорционален произведению адронной плотности вещества на мембране и пятимерной гравитационной постоянной. Это небольшое число, $\delta_{\mathrm{h}_{0}} \sim 10^{-9}$. Так как сталкиваются адроны с лоренцевским фактором $\gamma=$ $1 / \sqrt{1-v^{2}}$ порядка $\gamma \sim 10^{4}$, мы получаем $\delta_{\mathrm{h}} \sim 10^{-5}$. Для тяжелых ионов, состоящих из $A$ адронов, это число порядка

$$
\delta_{\text {ion }} \sim A_{\text {ion }}^{1 / 3} \delta_{\mathrm{h}}
$$

Можно сравнить это число с оценкой дефицита угла $\delta_{\mathrm{cs}} \sim 10^{-6}$ для космической струны в четырехмерном пространстве-времени с ньютоновской гравитационной постоянной $G_{\mathrm{N}, 4}$ и плотностью $\rho=m / l=10^{33}$ ГэВ ${ }^{2}$, которая соответствует массе, равной массе Земли, распределенной на длине около $L=9$ км.

2.2.2. Метрика в модели RS2 с конической особенностью. Деформацию гравитационного фона в модели RS2 за счет адронной мембраны удобно описывать в координатах Пуанкаре. Исходная метрика в этих координатах имеет вид

$$
d s^{2}=a^{2}(y) \eta_{\mu \nu} d x^{\mu} d x^{\nu}+d y^{2},
$$

где $\eta_{\mu \nu}$ - четырехмерная метрика Минковского, множитель $a(z)$ имеет вид [11]

$$
a(y)=e^{-k|y|},
$$

$1 / k$ - радиус пятимерного пространства-времени анти-де Ситтера. После замены переменных $y \rightarrow w, w=r_{0} e^{y / r_{0}}$ мы получаем метрику Пуанкаре [8]:

$$
d s^{2}=\frac{r_{0}^{2}}{w^{2}}\left(\eta_{\mu \nu} d x^{\mu} d x^{\nu}+d w^{2}\right) .
$$

Чтобы учесть мембрану, в соответствии с обычной процедурой мы удаляем клин, сечение образующих плоскостей которого дается дефицитом угла (см. рис. 3). Это может быть сделано за счет сокращения диапазона подходящих угловых координат. Например, для пространства $A d S_{5}$

$$
d s^{2}=\frac{r_{0}^{2}}{w^{2}}\left(d w^{2}-d t^{2}+d z^{2}+d \rho^{2}+\rho^{2} d \phi^{2}\right),
$$

и угол $\phi$ изменяется в пределах $0<\phi<2 \pi-\delta$, где $\delta$ определяется формулой (3). 


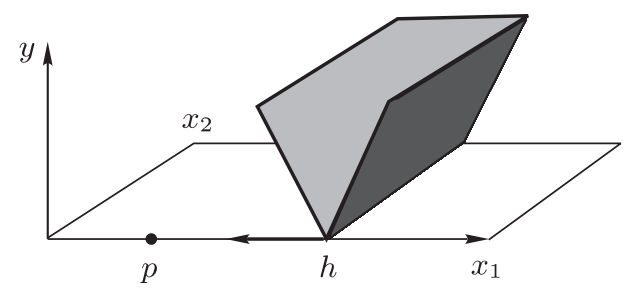

Рис. 3. Ультрарелятивистский адрон $h$, как его видит партон $p$ из налетающего адрона.

2.3. Анизотропная компактификация с $M_{\mathrm{Pl}, D} \sim 1 \mathrm{T \ni}$. Для анизотропной компактификации, когда размеры в одном направлении выбраны больше размеров других дополнительных координат, мы имеем следующее соотношение между $D$-мерной и четерехмерной массами Планка:

$$
\left(\frac{M_{\mathrm{Pl}, 4}}{M_{\mathrm{Pl}, D}}\right)^{2}=\left(\frac{M_{\mathrm{Pl}, D}}{M_{\mathrm{c}}}\right)^{D-5} \frac{M_{\mathrm{Pl}, D}}{M_{1}},
$$

где $M_{1}$ - масштаб выделенного одного измерения, $M_{\mathrm{c}}$ - масштаб дополнительных $N-1$ измерений. Формула (8) следует из обычной редукции $D$-мерного пространства до четырехмерного:

$$
\frac{1}{M_{\mathrm{Pl}, 4}^{2}} \int d^{4} x \sqrt{-g} R=\frac{V_{\mathrm{c}}}{M_{\mathrm{Pl}, D}^{D-2}} \int d^{4} x \sqrt{-g} R=\frac{1}{M_{\mathrm{Pl}, D}^{D-2}} \int d^{D} x \sqrt{-g} R
$$

и учета выбранного способа компактификации.

В случае

$$
M_{\mathrm{Pl}, D}=M_{\mathrm{SM}}=1 \mathrm{~T} \ni \mathrm{B},
$$

где $M_{\mathrm{SM}}-$ характерный масштаб Стандартной модели, мы получаем

$$
10^{32}=\frac{M_{\mathrm{Pl}, D}}{M_{1}}\left(\frac{M_{\mathrm{Pl}, D}}{M_{\mathrm{c}}}\right)^{D-5} .
$$

Считая, что характерные длины компактификации $l_{1} \sim 1 / M_{1}, l_{\mathrm{c}} \sim 1 / M_{\mathrm{c}}$ связаны c $l_{\mathrm{SM}} \approx 10^{-16} \mathrm{~cm} \mathrm{как}$

$$
l_{1}=10^{r_{1}} l_{\mathrm{SM}}, \quad l_{\mathrm{c}}=10^{r_{\mathrm{c}}} l_{\mathrm{SM}},
$$

из (11) мы получаем

$$
r_{1}=32-r_{\mathrm{c}}(D-5)
$$

Отметим, что мы также предполагаем выполнение неравенства $a>1$, где величина $a=r_{1}-r_{\mathrm{c}}$ связывает $r_{1}$ и $r_{\mathrm{c}}$, т. е. мы считаем, что параметр анизотропии $l_{1} / l_{\mathrm{c}} \geqslant 10$.

Из рис. 4 видно, что для того чтобы $r_{\mathrm{c}}$ было мало, мы должны рассматривать большое $D$. В частности, для изотропной компактификации, чтобы иметь масштаб компактификации меньше адронного масштаба $l_{\mathrm{h}} \sim 1 \Phi_{\mathrm{M}}=10^{-13} \mathrm{cм}$, мы должны взять $D=10$. 


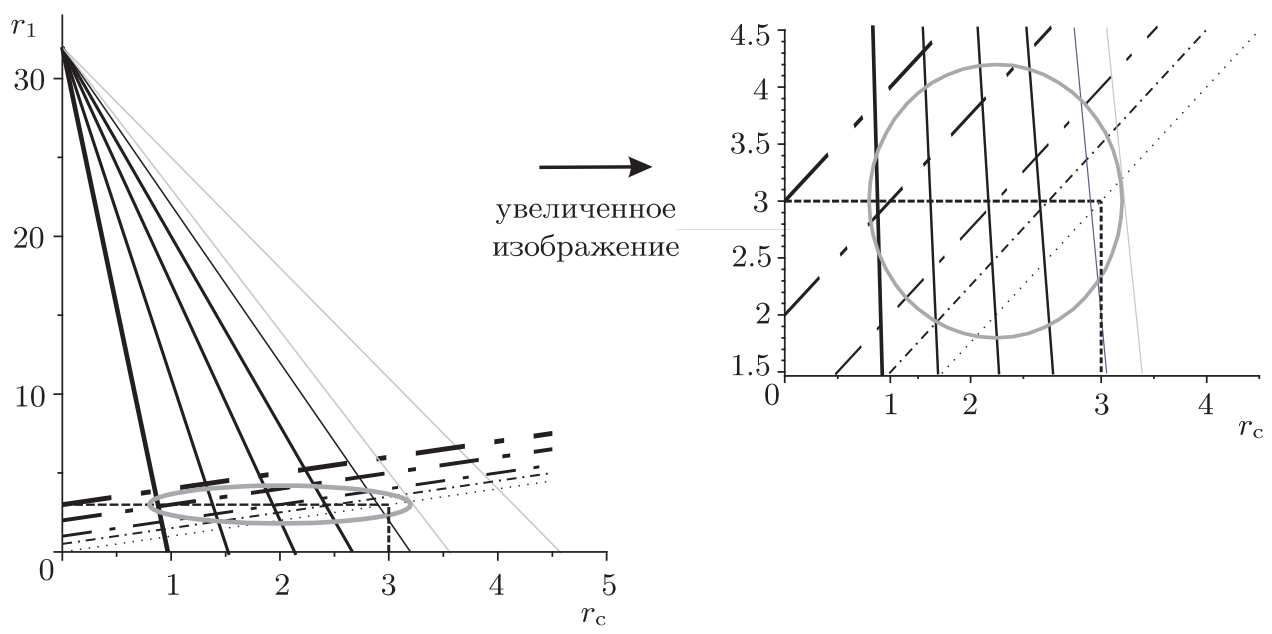

Рис. 4. Зависимость $r_{1}=r_{1}\left(r_{\mathrm{c}}\right)$ (13) для различных $D=12,14,15,17,20$, 26,38 изображена сплошными прямыми линиями (увеличение толщины соответствует увеличению $D$ ). Штриховые линии дают ограничения на масштаб адронов. Пунктирная линия $r_{1}=r_{\mathrm{c}}$ соответствует симметричной компактификации. Штрихпунктирные линии $r_{1}=r_{\mathrm{c}}+a, a=0.5,1,2,3$, соответствуют асимметричной компактификации (увеличение толщины соответствует увеличению параметра асимметрии $a)$.

Поскольку мы также предполагаем, что размеры адрона больше радиуса компактификации $l_{\mathrm{c}}$, мы получаем

$$
l_{1}<l_{\mathrm{h}}=10^{-13} \mathrm{cM}=10^{3} l_{\mathrm{SM}} \quad \Rightarrow \quad r_{1}<3,
$$

и для анизотропной компактификации с параметром анизотропии $a \sim 1$ мы должны взять $D \geqslant 14$. Предполагая меньший параметр анизотропии, скажем $a \sim 0.5$, т.е. $l_{1} \approx 3 l_{\mathrm{c}}$, мы можем рассматривать $D=13$. Большой параметр анизотропии

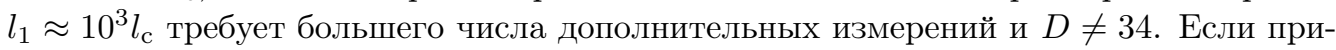
цельный параметр $b$ больше, чем радиус компактификации $n-1$ измерений, этими дополнительными измерениями можно пренебречь. В этом режиме можно было бы зондировать дополнительные измерения только с помощью гравитационных взаимодействий.

В этом случае мы можем оценить величину $M_{\mathrm{Pl}, 5}$ (так как именно эта постоянная должна быть принята во внимание во всех приведенных ниже расчетах):

$$
M_{\mathrm{Pl}, 5}^{3}=M_{\mathrm{Pl}, D}^{D-2}\left(\frac{1}{M_{1}}\right)^{D-5}
$$

или

$$
M_{\mathrm{Pl}, 5}^{3}=M_{\mathrm{SM}}^{D-2}\left(l_{\mathrm{c}}\right)^{D-5}=M_{\mathrm{SM}}^{D-2}\left(l_{\mathrm{SM}} 10^{r_{\mathrm{c}}}\right)^{D-5}=M_{\mathrm{SM}}^{3} 10^{r_{\mathrm{c}}(D-5)} .
$$

Таким образом, мы получаем

$$
M_{\mathrm{Pl}, 5}=M_{\mathrm{SM}} 10^{r_{\mathrm{c}}(D-5) / 3} .
$$


Выбирая $D=30, r_{\mathrm{c}}=1$, мы имеем

$$
M_{\mathrm{Pl}, 5}=M_{\mathrm{SM}} 10^{(25-5) / 3} \approx M_{\mathrm{SM}} 10^{6.8} .
$$

Такая гравитация не достаточно сильна, чтобы произвести заметные эффекты в наземных экспериментах. Более предпочтителен выбор $r_{\mathrm{c}}=0.3$ и $M_{\mathrm{Pl}, 5} \sim 10^{3}$ ТэВ.

\section{3. АМПЛИТУДЫ РАССЕЯНИЯ ЧАСТИЦ В ОКРЕСТНОСТИ АДРОННОЙ МЕМБРАНЫ}

Известно, что эффект присутствия космической струны эквивалентен присутствию внешнего поля, хотя гравитационного потенциала не возникает и эффект носит чисто топологический характер. Разумеется, аналогичный эффект происходит и в случе космической мембраны в пятимерном пространстве-времени.

Рассмотрим взаимодействие типа Юкавы, $V_{\text {int }}=\int d^{x} \lambda \phi \psi^{2}$, между легкой частицей $\Phi$ и тяжелой частицей $\Psi$ с массой $M$. Как и в случае космической струны [13], можно оценить распад легкой частицы на две тяжелые частицы с массой $M$ :

$$
\begin{aligned}
& \left\langle p, \vec{p}_{\mathrm{tr}}, n ; q, \vec{q}_{\mathrm{tr}}, m\left|V_{\mathrm{int}}\right| k, \vec{k}_{\mathrm{tr}}, l\right\rangle= \\
& \quad=\frac{\nu^{3 / 2} \lambda}{\sqrt{8 w E_{1} E_{2}}} \delta\left(w-E_{1}-E_{2}\right) \delta^{2}\left(\vec{k}_{\mathrm{tr}}-\vec{p}_{\mathrm{tr}}-\vec{q}_{\mathrm{tr}}\right) \delta_{l, m+n} A_{m, n}
\end{aligned}
$$

где

$$
A_{n, m}=\int d \rho \rho J_{|m+n| \nu}(k \rho) J_{|m| \nu}(q \rho) J_{|n| \nu}(p \rho) .
$$

Поясним введенное обозначение и отличия от случая космической струны. Индексы в этой формуле связаны с индексами, нумерующими решения уравнения Клейна-Гордона в пятимерном пространстве-времени в присутствии космической мембраны,

$$
\begin{aligned}
\Psi\left(y, x_{1}, \vec{x}_{\mathrm{tr}}, t\right)= & \int_{\mathbb{R}^{2}} d \vec{p}_{\mathrm{tr}} \int_{0}^{\infty} \sum_{n=-\infty}^{\infty} J_{|n| \nu}(p \rho) e^{i n \nu \theta} e^{i \vec{p}_{\mathrm{tr}} \vec{x}_{\mathrm{tr}}} \times \\
& \times \frac{\sqrt{\nu}}{2 \pi}\left[a_{n}\left(p, \vec{p}_{\mathrm{tr}}\right) \frac{e^{-i E t}}{\sqrt{2 E}} a_{n}^{+}\left(p,-\vec{p}_{\mathrm{tr}}\right) \frac{e^{-i E}}{\sqrt{2 E_{\Psi}}}\right]
\end{aligned}
$$

где $\Psi=\psi, \phi, E_{\Psi}=\sqrt{p^{2}+\vec{p}_{\mathrm{tr}}^{2}+M_{\Psi}^{2}}, \vec{x}_{\mathrm{tr}}=\left\{x_{2}, x_{3}\right\}$ и $\vec{p}_{\mathrm{tr}}=\left\{p_{2}, p_{3}\right\}-$ векторы вдоль космической мембраны, $\vec{p}_{l}$ - вектор в двумерной плоскости, ортогональной космической мембране, а $p=\left|\vec{p}_{l}\right|$. Компоненты импульсов вдоль мембраны сохраняются, а компоненты в ортогональном направлении не сохраняются (этот эффект аналогичен отсутствию сохранения пространственных компонент импульсов в $(1+2)$-мерной гравитации [14], [15]). По условию бранного сценария для частиц $\vec{p}_{l}=\left\{p_{1}, 0\right\}, n, m$ и $l$ - угловые моменты тяжелых $(\psi)$ и легкой $(\phi)$ частиц. В формуле (19) учтено, что угловой момент сохраняется, $l=n+m$.

Сумма по всем моментам выходящих частиц вычисляется явно:

$$
\sum_{n, m} A_{n, m}^{2} \delta_{l, n+m}=\frac{4 \sin ^{2} \pi \nu}{\pi} \mathcal{F}_{l}(p, q, k) .
$$


Функция $\mathcal{F}_{l}(p, q, k)$ совпадает с ответом для космической струны

$$
\begin{aligned}
F_{l}(s, t) & =\frac{\left(a^{|l|}+b^{|l|}\right) a b(1+a b)}{(1-a b)\left[(1-a b)^{2}+4 a b \sin ^{2} \pi \nu\right]}, \\
a & =\left(\frac{1+r t-s}{1+r t+s}\right)^{\nu}, \quad b=\left(\frac{1-r t-s}{1-r t+s}\right)^{\nu}, \\
r & =\frac{p^{2}+q^{2}}{k^{2}}, \quad t=\frac{p^{2}-q^{2}}{p^{2}+q^{2}}, \quad s^{2}=1-2 r+r^{2} t^{2} .
\end{aligned}
$$

Выражение для сечения на единицу площади мембраны имеет вид

$$
\sigma_{l}=\frac{\nu \lambda^{2} \sin ^{2} \pi \nu}{32 \pi^{6} w k^{4}} f(p, q, r) \mathcal{F}_{l}(p, q, k),
$$

где $f(p, q, r)$ для мембраны определяется двумерными интегралами:

$$
f(p, q, r) \equiv \int d^{2} \vec{p}_{\mathrm{tr}} \int d^{2} \vec{q}_{\mathrm{tr}} \frac{\delta\left(w-E_{1}-E_{2}\right)}{E_{1} E_{2}} \delta^{2}\left(\vec{k}_{\mathrm{tr}}-\vec{p}_{\mathrm{tr}}-\vec{q}_{\mathrm{tr}}\right) .
$$

Функция $f(p, q, r)$ приводится к виду

$$
f(p, q, r)=\frac{4 \pi}{k} \theta\left(s^{2}-4 \frac{M^{2}}{k^{2}}\right) .
$$

Окончательный ответ имеет вид

$$
\sigma_{l}=\frac{\nu \lambda^{2} \sin ^{2} \pi \nu}{32 \pi^{5} w k} \int_{0}^{1} d t \int_{2 M / k}^{1} d s \frac{\left(1-s^{2}\right) F_{l}(s, t)}{s\left[1-t^{2}\left(1-s^{2}\right)\right]^{1 / 2}\left[1+\left(1-t^{2}\left(1-s^{2}\right)\right)^{1 / 2}\right]},
$$

что отличается от соответствующего соотношения для струны.

Однако, как и для струны, в случае большого продольного импульса легкой частицы, $k_{z} \gg 2 M \delta^{-1}$, сечение не зависит от $k_{z}$ и определяется константой связи $\lambda$ :

$$
\sigma_{1 \text { light } \rightarrow 2 \text { heavy }} \approx \frac{\lambda^{2}}{M^{3}}
$$

Для реализации условия $k_{z} \gg 2 M \delta$ достаточно, чтобы $k_{z} \sim 1$ ТэВ и масса $M$ составляла порядка нескольких МэВ.

Благодарности. Автор благодарна И. Воловичу, А. Радюшкину и А. Багрову за плодотворные обсуждения. Работа была частично поддержана РФФИ (гранты № 08-01-00798, 09-01-12179-офи_м) и Программой поддержки ведущих научных школ (грант НШ-8265-2010.1).

\section{Список литературы}

[1] N. Arkani-Hamed, S. Dimopoulos, G. Dvali, Phys. Lett. B, 429:3-4 (1998), 263-272, arXiv: hep-ph/9803315.

[2] I. Antoniadis, N. Arkani-Hamed, S. Dimopoulos, G. R. Dvali, Phys. Lett. B, 436:3-4 (1998), 257-263, arXiv: hep-ph/9804398.

[3] В. А. Рубаков, М. Е. Шапошников, УФН, 166 (1996), 493-537, arXiv: hep-ph/9603208. 
[4] T. Banks, W. Fischler, A model for high energy scattering in quantum gravity, arXiv: hep-th/9906038.

[5] I. Ya. Aref'eva, ЭЧАЯ, 31:7a (2000), 169-180, arXiv: hep-th/9910269.

[6] I. Ya. Aref'eva, ЭЧАЯ, 41:6 (2010), 1562-1578, arXiv: 0912.5481.

[7] I. Ya. Aref'eva, Colliding hadrons as cosmic membranes and possible signatures of lost momentum, arXiv: 1007.4777.

[8] A. Vilenkin, Phys. Rep., 121:5 (1985), 263-315.

[9] S. Deser, R. Jackiw, G. 't Hooft, Ann. Phys., 152:1 (1984), 220-235.

[10] S. Holst, H.-J. Matschull, Class. Quantum Grav., 16:10 (1999), 3095-3131, arXiv: gr-qc/9905030.

[11] L. Randall, R. Sundrum, Phys. Rev. Lett., 83:17 (1999), 3370-3373, arXiv: hep-ph/9905221.

[12] G. Dvali, G. Gabadadze, M. Porrati, Phys. Lett. B, 485:1-3 (2000), 208-214, arXiv: hep-th/0005016.

[13] V.D. Skarzhinsky, D. D. Harari, U. Jasper, Phys. Rev. D, 49:2 (1994), 755-762, arXiv: astro-ph/9311029.

[14] M. Henneaux, Phys. Rev. D, 29:12 (1984), 2766-2768.

[15] S. Deser, Class. Quantum Grav., 2:4 (1985), 489-495.

Поступила в редакцию 6.09.2010 